\title{
Magnetorheological behavior of magnetite covered clay particles in aqueous
}

\section{suspensions.}

\author{
C. Galindo-Gonzalez, ${ }^{1 \text { a.b) }}$ M.T. Lopez-Lopez, ${ }^{1}$ J.D.G. Duran ${ }^{1}$ \\ ${ }^{1}$ Department of Applied Physics, University of Granada, Avda Fuentenueva s/n, 18071, \\ Granada, Spain.
}

Montmorillonite clay particles coated with magnetite nanoparticles suspended in aqueous media behave as magnetorheological fluids with enhanced stability as compared to conventional ones. In this work the study of the magnetorheological behavior of these suspensions of magnetite-clay composite particles has been carried out. For this purpose both steady and dynamic rheological measurements were carried out in the absence and in the presence of external magnetic fields. In the first kind of experiments the rheograms of the suspensions (shear stress versus shear rate plot) are analyzed as a function of the strength of the magnetic field applied. In the second one, oscillatory stresses are applied to the system and the storage modulus is studied as a function of the external magnetic field. In the absence of magnetic field, the suspensions develop a weak yield stress due to the aggregation of the magnetite covered clay particles. In the presence of magnetic field, the yield stress is strongly dependent on the magnetic field strength inside the samples, demonstrating that the suspensions experience a magnetorheological effect, moderate when the magnetic field strength is weak and stronger for values of magnetic field higher than

\footnotetext{
a) Author to whom correspondence should be addressed. Electronic mail: cgalindo@ugr.es

b) Present address: Laboratoire Matiere et Systemes Complexes, Univesité Paris Diderot, Sorbonne Paris Cité-Paris7, 10 rue Alice Domont et Léonie Duquet 75205 Paris cedex 13.
} 
$150-200 \mathrm{kA} / \mathrm{m}$. This change of regime for the dependence of the yield stress with the field shows up fitting the $\sigma_{\mathrm{y}}=\sigma_{\mathrm{y}}(\mathrm{H})$ curve: two different power laws are used for the values of magnetic field strength smaller and higher than $150-200 \mathrm{kA} / \mathrm{m}$. The results are interpreted by means of a model that relates the structure of the particles in the suspensions to the magnetic field applied and using the interaction energy between particles calculated by the extended DLVO theory to include magnetic interaction.

\section{INTRODUCTION}

Conventional magnetic fluids are composed of ferro or ferrimagnetic particles dispersed in a carrier liquid that can be aqueous or not aqueous. Their main feature is that their rheological behavior can be controlled by means of external magnetic fields. These are capable of making the fluid to undergo a transition from a Newtonian liquid when there is not a magnetic field, to a plastic fluid when a magnetic field is applied. This transition, called magnetorheological (MR) effect ${ }^{1,2}$ is due to a process of particle chaining favored by the magnetic attraction between the magnetic dipoles induced by the field.

The above mentioned characteristic has promoted the use of these materials in technological applications, such as controllable dampers, shock absorbers, brakes and others. ${ }^{3,4}$

The MR effect of a magnetic fluid depends on several features, as the size, shape, composition, and volume fraction of the dispersed particles, and the strength of the applied magnetic field. The main properties of magnetic fluids are the yield stress (minimum value of the applied stress that provokes the flow of the suspension), the viscosity and the rate and degree to which the particles settle. ${ }^{2}$ There are different ways to enhance the MR effect. An increase in the volume fraction of the particles in the suspension is one of these ways, as it usually provokes an increase in the field- 
induced yield stress. But a rise in the volume fraction of the particles also increases the viscosity of the suspension in absence of external magnetic field and the degree of settling of the particles in the suspension.

The size of the particles plays an important role in the behavior of the fluid. On one hand, micron-sized particles provide a higher MR response, ${ }^{5}$ but as the particles are bigger, the sedimentation becomes more important and particles larger than about $10 \mu \mathrm{m}$ tend to settle rapidly even with the addition of additives. On the other hand, nanoparticles settle more slowly and sometimes are even stable against sedimentation, but they give rise to smaller MR effect.

Moreover, in most of the magnetic fluids the sedimentation of the particles occurs due to the difference in density between the particles and the carrier liquid. Once settled, the particles form packed sediments caused by remanent magnetization and they are not easily redispersed ${ }^{2}$. In order to avoid the settling of the particles and the formation of the sediments, both the particles and the carrier liquid can be modified. The addition of special additives, such as surfactants, nanoparticles and other particles has been studied in order to overcome the problems associated with settling. ${ }^{6-}$ ${ }^{12}$ Changes in the shape of the particles ${ }^{7,10-21}$ or adding of elongated ferromagnetic particles to magnetic fluids of spherical particles ${ }^{7,22}$ have been explored also to reduce the sedimentation.

In previous works ${ }^{23,24}$ we studied the aggregation and sedimentation behavior of magnetic fluids consisting of aqueous suspensions of magnetic particles composed by a clay core of sodium montmorillonite covered by a shell of magnetite nanoparticles. For diluted suspensions, ${ }^{23}$ we proved that the sedimentation rates of the particles are slower than those obtained in suspensions of micron-sized magnetic particles. In the case of concentrated suspensions of magnetite-covered clay particles, the very slow gravitational settling makes these fluids behave as stable magnetic 
fluids because they do not suffer of the usual phase separation observed in suspensions of pure ferri or ferromagnetic micron-sized particles.

In order to check if these fluids can be considered as a real alternative for formulating magnetic fluids in which the settling and redispersion difficulties are reduced, their magnetorheological behavior has to be analyzed, which is the aim of the present work.

\section{EXPERIMENTAL DETAILS}

\section{A. Materials}

The sodium montmorillonite (NaMt) particles were obtained by means of a homoionization procedure from a natural bentonite extracted from a deposit located in the southeastern region of the Spanish peninsula (Almería, Spain). The homoionization process, specific surface area (54.1 $\mathrm{m}^{2} / \mathrm{g}$ ), surface and bulk chemical composition, and X-ray diffraction pattern were reported elsewhere. $^{23,25}$ The NaMt particles have a plate-like shape with a length of approximately $1 \mu \mathrm{m}$ and an aspect ratio (particle equivalent diameter/thickness) of about $14 .^{26}$

Magnetite nanoparticles were synthesized by the co-precipitation method proposed by Massart, ${ }^{27}$ which consists of the following steps: $(i)$ a solution of ferrous chloride $(10 \mathrm{~mL}, 2 \mathrm{~mol} / \mathrm{L}$ $\mathrm{FeCl}_{2}$ in $2 \mathrm{~mol} / \mathrm{L} \mathrm{HCl}$ solution) was mixed with a ferric chloride solution $(40 \mathrm{~mL}, 1 \mathrm{~mol} / \mathrm{L})$ in alkaline medium $\left(500 \mathrm{~mL}, 0.7 \mathrm{~mol} / \mathrm{L} \mathrm{NH} \mathrm{NH}_{3}\right.$ solution) under continuous mechanical stirring; (ii) stirring was maintained during $20 \mathrm{~min}$; (iii) the obtained solid particles were settled by magnetic decantation by means of a permanent magnet $(B=0.42 \mathrm{~T})$ and the remaining ammonia solution was discarded; (iv) then, the particles were redispersed in $2 \mathrm{~mol} / \mathrm{L} \mathrm{HClO}_{4}$ aqueous solution; (v) after a few hours, the perchloric acid solution was discarded after magnetic decantation of the 
magnetite particles and, finally, the magnetite was redispersed in aqueous solution. The magnetite particles were approximately spherical, as observed by high resolution transmission electron microscopy, and their average diameter was $11.1 \pm 2.2 \mathrm{~nm}^{23}$ The specific surface area of the powder was $42.2 \mathrm{~m}^{2} / \mathrm{g}$ as determined by $\mathrm{N}_{2}$ adsorption using the BET multipoint method in a Quantasorb Jr. apparatus (Quantachrome, USA). The magnetization, $M$, of the magnetite nanoparticles was measured at $293.0 \mathrm{~K}$ as a function of the applied magnetic field strength, $H$, in a Manics DSM-8 magnetosusceptometer (France).

Deionized and filtered water (Milli-Q Academic, Millipore, France) was used in the preparation of all the solutions and suspensions. The chemicals used were purchased from either SigmaAldrich (Germany) or Panreac (Spain) with analytical quality, and were not further purified.

\section{B. Preparation of the suspension}

The samples used for the study of the rheological properties of the system were suspensions with a clay volume fraction $\phi_{C}=3 \%$ and a magnetite to clay volume fraction ratio $\phi_{M} / \phi_{C}=3$. The preparation of the suspensions was carried out by means of the following procedure: $(i)$ two clay and magnetite stock suspensions were prepared separately in $2 \times 10^{-3} \mathrm{M} \mathrm{NaNO}_{3}$ solution and their $\mathrm{pH}$ adjusted at $\mathrm{pH}=3$; $(\mathrm{ii})$ proper amounts of clay and magnetite stock suspensions were mixed in order to reach the desired $\phi_{M} / \phi_{C}$ ratio and the $\mathrm{pH}$ was readjusted at $\mathrm{pH}=3$.

As demonstrated in a previous work, ${ }^{23}$ this procedure ensures that the clay and magnetite particles bear opposite surface charge (clay negative, magnetite positive) at the $\mathrm{pH}$ and ionic strength of the stock suspensions. After mixing the stock suspensions, the coverage of the clay particles by the magnetite ones was favored by the electrostatic attraction between them. The formation of clay-magnetite composite entities was demonstrated by carrying out a 
characterization of the resulting solid particles and suspensions that included the magnetic properties, electron microscopy pictures, and electrophoretic measurements [see Ref 23].

\section{Methods}

The rheological measurements were performed in a controlled rate magnetorheometer (MCR 300, Anton Paar-Physica, Germany) with parallel-plate geometry (diameter $20 \mathrm{~mm}$; gap $0.35 \mathrm{~mm}$ ), at least $24 \mathrm{~h}$ after the preparation of the suspensions. The magnetic field was applied by means of a coil placed below the bottom plate of the rheometer, which gives a magnetic field strength $H_{0}=$ $343 \mathrm{kA} / \mathrm{m}$ in the air gap between the plates for the maximum electric current applied (2 A).

In order to characterize the rheological properties of the suspension two kinds of measurements were performed:

(i) Steady-state regime measurements: the samples were subjected to a shear rate ramp between $10^{-4}$ and $1000 \mathrm{~s}^{-1}$ and the corresponding shear stress and dynamic viscosity were recorded as a function of the shear rate. This kind of plot is called rheogram. The applied shear ramp was logarithmic, the number of data recorded was 60 and the time between each of them was 5 seconds.

(ii) Dynamic rheological experiments: A ramp of sinusoidal stress with constant frequency $f=1 \mathrm{~Hz}$ and variable amplitude between 0.1 and $100 \mathrm{~Pa}$ was applied and the storage modulus, G', and the loss modulus, G', were obtained as a function of the stress amplitude. The applied stress ramp was logarithmic, the number of data recorded was 40 and the time between each of them was 15 seconds. The storage modulus, $G^{\prime}$, is the real part of the complex rigidity modulus, $G^{*}$, that relates the sinusoidal stress and the sinusoidal strain, both in their complex form. $G^{\prime}$ is proportional to the storage power per volume unity in the suspension during a quarter of cycle. ${ }^{28}$ The complex part, $G$ ', 
of the rigidity modulus is called loss modulus and it is proportional to the dissipated power by viscous friction.

TABLE I. Magnetic flux density and magnetic field strengths corresponding to the electric current applied in the coil of the magnetorheometer in the different measurements.

\begin{tabular}{cccccc}
\hline \hline $\mathrm{I}(\mathrm{A})$ & $\mathrm{B}(\mathrm{mT})$ & $\mathrm{H}(\mathrm{kA} / \mathrm{m})$ & $\mathrm{I}(\mathrm{A})$ & $\mathrm{B}(\mathrm{mT})$ & $\mathrm{H}(\mathrm{kA} / \mathrm{m})$ \\
\hline 0.1 & 13.7 & 10.9 & 1.1 & 216 & 171.9 \\
0.2 & 31 & 24.7 & 1.2 & 235.5 & 187.4 \\
0.3 & 51 & 40.6 & 1.3 & 254.1 & 202.2 \\
0.4 & 71.6 & 57 & 1.4 & 271.6 & 216.1 \\
0.5 & 92.3 & 73.5 & 1.5 & 287.9 & 229.1 \\
0.6 & 113.5 & 90.3 & 1.6 & 303.2 & 241.3 \\
0.7 & 134.5 & 107 & 1.7 & 317.3 & 252.5 \\
0.8 & 155.2 & 123.5 & 1.8 & 330.4 & 262.9 \\
0.9 & 175.6 & 139.7 & 1.9 & 342.8 & 272.8 \\
1 & 196 & 156 & 2 & 354.5 & 282.1 \\
\hline \hline
\end{tabular}

In order to ensure the reproducibility of the experiments in both sets of measurements, before the application of the shear ramp, a pre-shear in the post-yield regime was applied during $20 \mathrm{~s}$, followed by an equilibrium time of $60 \mathrm{~s}$. The magnetic field was introduced at the end of the preshear step and was maintained along the whole equilibrium time and sweeps.

Different measurements with electric current applied from 0 to $2 \mathrm{~A}$ with a step of $0.1 \mathrm{~A}$ between them were carried out. The magnetic field strengths corresponding to these currents are displayed in Table I.

\section{RESULTS AND DISCUSION}

\section{A. Magnetic properties}

Figure 1 shows the magnetization curve of the synthetic magnetite powder. As observed, a saturation magnetization $M_{S} \approx 427 \mathrm{kA} / \mathrm{m}$ was obtained. This value is lower than that obtained for 
micron-sized synthetic magnetite $\left(M_{s}=570.7 \mathrm{kA} / \mathrm{m}\right)^{29}$ and, as expected, larger than that of micronsized cobalt ferrite particles $\left(M_{S}=270 \mathrm{kA} / \mathrm{m}\right)^{30}$. The value reported in the literature for pure magnetite is $M_{S}=510 \mathrm{kA} / \mathrm{m}^{31}$.

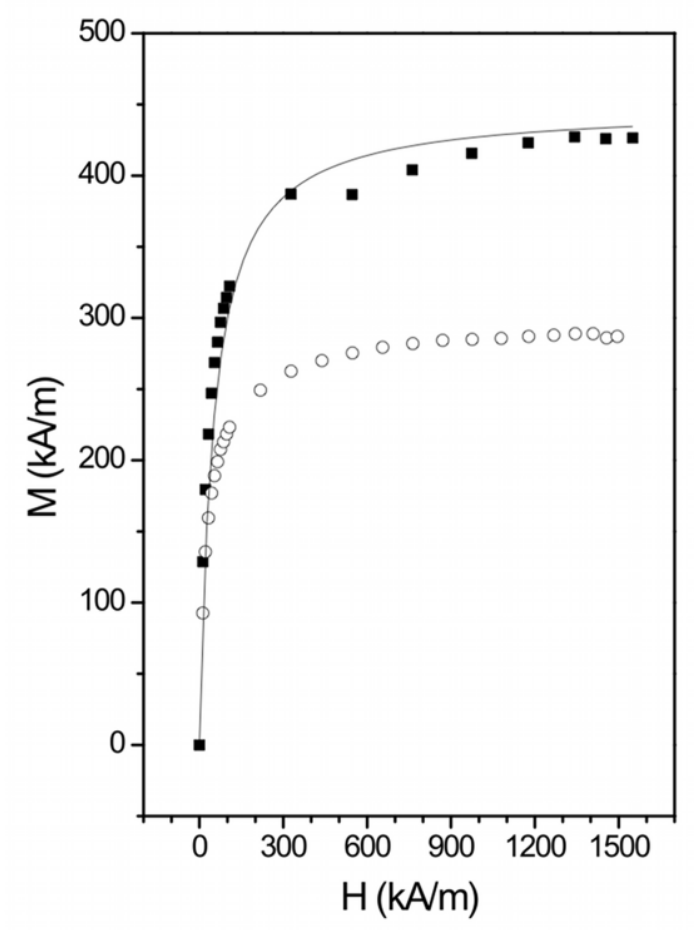

FIG 1. Magnetization curve of magnetite nanoparticles ( - ) and magnetite-covered clay particles with $\phi_{M} / \phi_{C}=3(0)$. Continuous line corresponds to the Fröhlish-Kennelly law fitting

In Figure 1 the magnetization curve for the magnetite-covered clay particles with $\phi_{M} / \phi_{C}=3$ is plotted as well. In this case, the saturation magnetization is lower than the corresponding to the magnetite particles.

More information about the magnetic properties of the synthesized magnetite nanoparticles and magnetite-covered clay particles can be found in previous works. ${ }^{23,32}$

\section{B. Interaction energy between particles}


In order to study deeply the rheological behavior of the suspensions, the analysis of the interaction energy between particles by means of the extended DLVO theory will be necessary. This theory takes into account electrostatic (EL), Lifshitz-van der Waals (LW), and hydration or acid-base $(\mathrm{AB})$ interactions. ${ }^{33,34}$

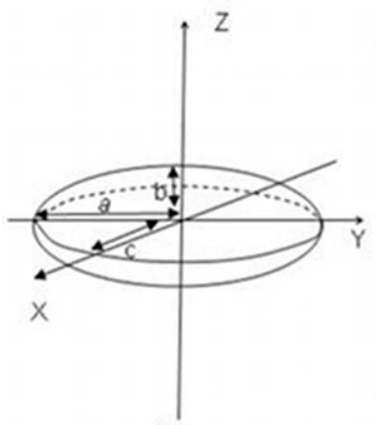

(a)

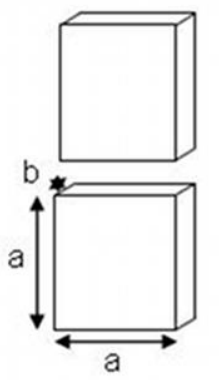

(c)

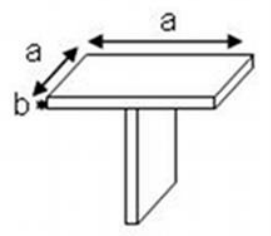

(b)

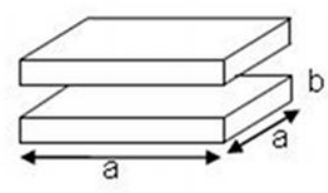

(d)

FIG 2. Schematic drawing of the (a) oblate spheroid geometry assumed for magnetite-covered clay particles. (b), (c), and (d) different orientations between particles: face-to-edge (b), edge-to-edge (c), and face-to-face (d).

Considering the plate-like geometry of the magnetite-covered clay particles, three possible orientations exist between them: face-to-face (F-F), face-to-edge (F-E), and edge-to-edge (E-E). The energy of interaction between two similar surfaces (F-F, E-E, F-E) (phase 1) separated by a surface-to-surface distance $h$, and immersed in an aqueous medium (phase 2) of moderate ionic strength can be calculated, assuming moderate surface potentials, by: ${ }^{33,34,35}$

$V_{121}^{E L}=\frac{\varepsilon \kappa}{8 \pi} 2 \zeta^{2}[1-\operatorname{coth}(\kappa h)+\operatorname{cosech}(\kappa h)]$ 


$$
\begin{aligned}
& V_{121}^{L W}=-\frac{A_{121}}{12 \pi h^{2}} ; A_{121}=24 \pi h_{0}^{2}\left(\sqrt{\gamma_{1}^{L W}}-\sqrt{\gamma_{2}^{L W}}\right) \\
& V_{121}^{A B}=V_{121}^{A B}\left(h_{0}\right) \exp \left(\frac{h-h_{0}}{\lambda}\right) ; V_{121}^{A B}\left(h_{0}\right)=-4\left(\sqrt{\gamma_{1}^{+} \gamma_{1}^{-}}+\sqrt{\gamma_{3}^{+} \gamma_{3}^{-}}-\sqrt{\gamma_{1}^{-} \gamma_{3}^{+}}-\sqrt{\gamma_{1}^{+} \gamma_{3}^{-}}\right)
\end{aligned}
$$

where $\varepsilon$ is the dielectric constant of the medium, $\kappa$ the reciprocal Debye length, $A_{121}$ the Hamaker constant, $h_{0}(0.158 \mathrm{~nm})$ the minimum separation distance between surfaces (for $h<h_{0}$, strong Born repulsion would appear), $\gamma_{i}^{j}$ the surface free energy components of the solid surface (see Table II) and the surface tension components of water $\left(\gamma_{3}^{L W}=21.8 \mathrm{~mJ} / \mathrm{m}^{2} ; \gamma_{3}^{+}=\gamma_{3}^{-}=25.5 \mathrm{~mJ} / \mathrm{m}^{2}\right)$, and $\lambda$ the correlation length of water molecules ( $\lambda=1 \mathrm{~nm}$ for hydrophilic surfaces).

TABLE II. Surface free energy components (in $\mathrm{mJ} / \mathrm{m}^{2}$ ) of magnetite and sodium montmorillonite (NaMt) obtained from contact angle measurements. $\gamma^{{ }^{W}}$ : Lifshitz-van der Waals; $\gamma^{+}$: electron-acceptor; $\gamma$ : electron-donor.

$$
\gamma^{W_{\mathrm{a}}} \quad \gamma^{+\mathrm{b}} \quad \gamma^{\mathrm{c}}
$$

\begin{tabular}{cccc}
\hline Magnetite & $49.3 \pm 0.2$ & $0.17 \pm 0.01$ & $55.4 \pm 0.3$ \\
NaMt & $44.2 \pm 0.4$ & $0.0 \pm 0.1$ & $60.6 \pm 0.5$
\end{tabular}

a $\gamma^{L W}$ is the Lifshitz-van der Waals component

${ }^{\mathrm{b}} \gamma^{+}$is the electron-acceptor component

${ }^{\mathrm{c}} \gamma$ is the electron-donor component

The equations (1-3) give the total energy per unit surface area $V_{121}^{T O T}\left(\mathrm{~J} / \mathrm{m}^{2}\right)$, but to obtain the total interaction energy $V^{T O T}$ (in Joule or better in $k T$ units, $k$ Boltzmann constant, $T$ temperature) $V_{121}^{T O T}$ must be multiplied by the estimated area of the clay-magnetite faces and edges. The surface area of faces $\left(S_{F}\right)$ and edges $\left(S_{E}\right)$ can be estimated as $S_{F} \approx a^{2}$ and $S_{E} \approx b^{2}$ (see Figure 2). In Figure 3 the total energy of interaction between the clay-magnetite particles $\left(\phi_{M} / \phi_{C}=3\right)$ as a function of the surface-surface separation is represented for the three orientations. At short distances between the particles $(\sim 10 \mathrm{~nm})$, both the electrostatic and acid-base interactions are repulsive, while the 
van der Waals one is attractive. As observed in Figure 3, whatever the relative orientation between the particles, a potential well exists for $h \approx 10 \mathrm{~nm}$ that favours aggregation in the suspension. Due to the larger surface area and the lower axis length, the most favorable aggregation process corresponds to the face-to-face orientation.
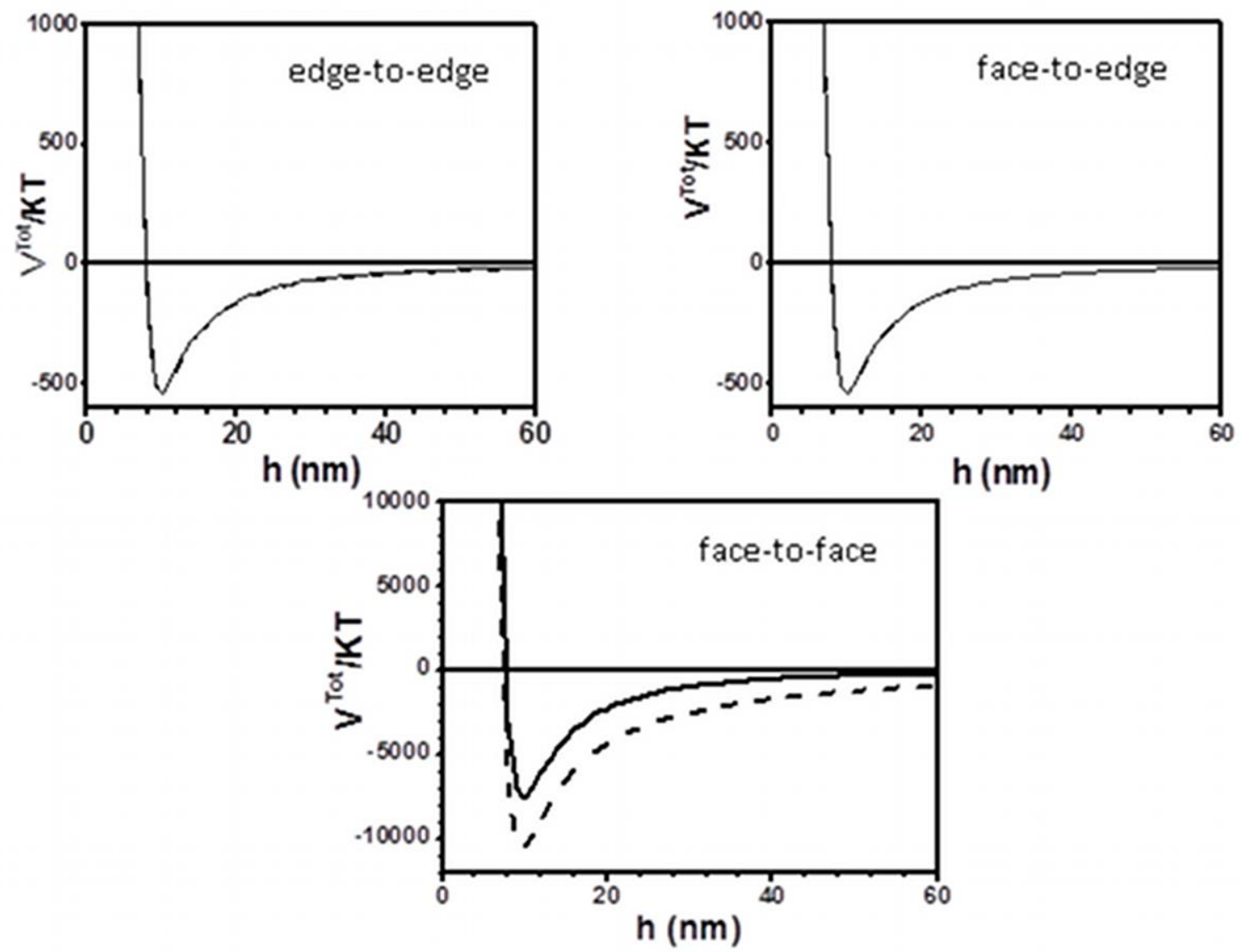

Figure 3 Total interaction energy between magnetite covered clay particles for the three different orientations as a function of the surface-to-surface distance $h$. Solid lines: without magnetic interaction energy. Dashed lines: considering the magnetic interaction energy (magnetic field strength used for the calculation $\mathrm{H}=10.9 \mathrm{kA} / \mathrm{m}$ )

On the other hand, when an external magnetic field is applied, the magnetic interaction energy between the particles has to be considered. For this reason the DLVO theory is extended to include magnetic interaction between aggregates. 
As a first step to calculate the magnetic interaction energy, the magnetic moment of the magnetite-covered clay particles has to be estimated. The magnetic moment of an isolated particle, of relative magnetic permeability $\mu_{p}$ surrounded by a medium of relative permeability $\mu_{m}$ and placed in an external magnetic field is: ${ }^{36}$

$$
\vec{m}=\mu_{0} V_{p} \frac{\mu_{p}-1}{\mu_{m}-N\left(\mu_{p}-1\right)} \vec{H}_{0}
$$

where $\mu_{0}$ is the vacuum permeability and $V_{p}$ the volume of the particle. $N$ is the demagnetizing factor that depends on the particle shape and the relative orientation of the particle in the external field, $\vec{H}_{0}$.

Taking into account the plate-like geometry of the clay core in the magnetite-covered clay particles, the composite particles can be supposed as oblate spheroids. The relative permeability of these particles can be calculated by means of: ${ }^{37}$

$$
\mu_{p}=1+\frac{\phi \beta}{1-\phi N \beta}
$$

where $\phi$ is the volume fraction of magnetite in the magnetite-clay composite particles, and $\beta$ is the magnetic contrast factor that, taking into account that the clay particles have a relative magnetic permeability equal to one, is given by:

$$
\beta=\frac{\mu_{p m}-1}{1+N\left(\mu_{p m}-1\right)}
$$

being $\mu_{p m}$ the relative magnetic permeability of the magnetite nanoparticles. The value of $\mu_{p m}$ is obtained by means of the magnetization curve of the magnetite particles shown in Figure 1. The magnetization data can be fitted by the Fröhlish-Kennelly law: ${ }^{38}$

$$
M=\frac{\chi_{i} H}{1+\chi_{i} / M_{S} H}
$$


where $\chi_{i}$, the magnetic susceptibility at $\mathrm{H} \rightarrow 0$, and $M_{S}$ the saturation magnetization, are the fitting parameters. The values corresponding to the best fit are $M_{S}=448 \mathrm{kA} / \mathrm{m}$ and $\chi_{i}=9.03$. Then, $\mu_{p m}$ $=\chi_{I}+1=10.03$. This $M_{S}$ and $\mu_{p m}$ values are well into the range giving in literature for magnetite solid particles. ${ }^{29,39}$

The demagnetizing factor for an oblate spheroid having two long axes $k$ times the length of the axis of symmetry, and magnetized parallel to a long axis is given by: ${ }^{40,41}$

$N_{x}=N_{y}=\frac{1}{2}\left\{\frac{k^{2}}{\left[k^{2}-1\right]^{3 / 2}} \arcsin \left(\frac{\left(k^{2}-1\right)^{1 / 2}}{k}\right)-\frac{1}{k^{2}-1}\right\}$

and for an oblate spheroid magnetized along its short axis:

$N_{z}=1-2 N_{x}=\frac{k^{2}}{k^{2}-1}\left\{1-\frac{1}{\left(k^{2}-1\right)^{1 / 2}} \arcsin \left(\frac{\left(k^{2}-1\right)^{1 / 2}}{k}\right)\right\}$

In order to estimate the axis ratio, $k$, the size of the magnetite-covered clay particles is calculated considering the size and density of the clay and magnetite particles and the volume fraction ratio between them in the composite particles.

Once the magnetic moment of the particles is calculated by means of Eq. 4, the magnetic interaction energy can be determined by:

$E_{d d}=\frac{1}{4 \pi \mu_{0}}\left[\frac{\vec{m}_{1} \cdot \vec{m}_{2}}{r^{3}}-\frac{3}{r^{5}}\left(\vec{m}_{1} \cdot \vec{r}\right)\left(\vec{m}_{2} \cdot \vec{r}\right)\right]$

where $\vec{m}_{1}$ and $\vec{m}_{2}$ are the magnetic moments of two different magnetite-covered clay particles, and $r$ is the distance between the centers of the particles. Considering that the magnetic moments are parallel and oriented head-to-tail, the equation can be rewritten: 
$E_{d d}=-\frac{m_{1} m_{2}}{2 \pi \mu_{0} r^{3}}$

Figure 3 shows the total energy of interaction between magnetite covered clay particles with $\phi_{M} / \phi_{C}=3$ as a function of the surface-to-surface distance, $\mathrm{h}$, for the three possible orientations, adding the magnetic interaction to the EL, LW and AB contributions (dashed lines). The value of the magnetic field strength used to calculate the magnetic interaction shown in Figure 3 is $\mathrm{H}=$ $10.9 \mathrm{kA} / \mathrm{m}$. In the case of face-to-edge and edge-to-edge orientations, the magnetic interaction is much lower than the sum of the other contributions. Thus, the total interaction energy does not change with the addition of the magnetic one. However, in the face-to-face orientation, the attractive magnetic interaction is comparable to the others. Hence, the total interaction becomes more attractive and the aggregation between particles in this orientation is more probable when an external magnetic field is applied.

\section{Steady state measurements. Yield stress}

Figure 4 shows the rheograms obtained for the different magnetic field strengths. The shape of the curves indicates that the suspension shows a plastic behavior, not apparent in the absence of the external magnetic field, when the suspension behaves almost like a Newtonian fluid. In reality, a small yield stress (minimum value of the stress that provokes the flow of the suspension) due to the aggregation of the magnetite covered clay particles can be observed even in the absence of external magnetic field.

One of the main features of the plastic behavior is the existence of a yield stress (minimum value of the applied stress that makes the suspension to flow). In the case of magnetic suspensions two different yield stresses are usually considered. The first one is the static yield stress, which is the minimum value of the stress for breaking the junction between the chains of particles (or the 
structure formed by the particles due to the external field) and the upper plate of the rheometer. The second one is the dynamic yield stress, which is the stress required to destroy the structures that are rebuilt in the presence of the applied field. The static yield stress can be estimated as the value of stress corresponding to the plateau of the rheogram in logarithmic scale, and the dynamic yield stress is the origin ordinate of the lineal fit of the last points of the rheogram in linear scale.

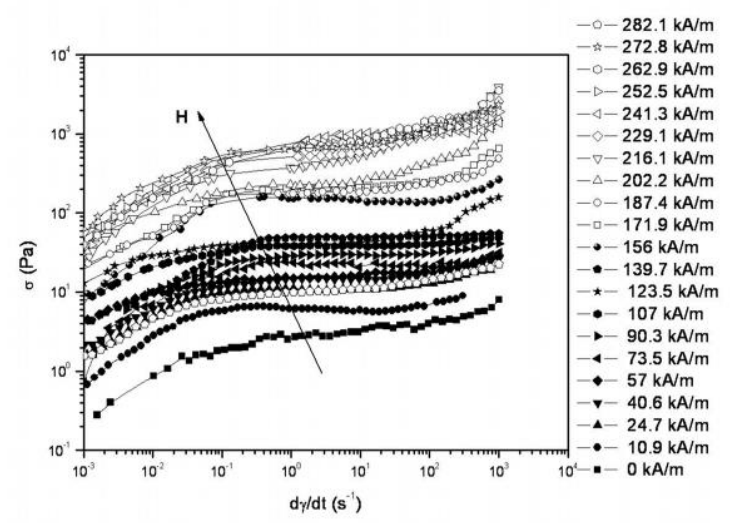

FIG 4. Rheograms of the suspension for the different magnetic fields applied. The strengths of the magnetic field corresponding to each curve are shown in the legend

In the case of the magnetite-clay composite particles, we have considered the static yield stress, because it is the yield stress used in the theoretical models that we consider. The estimation of the static yield stress (considered as the stress corresponding to the intermediate plateau of the curves) allows us the quantitative analysis of the magnetorheological behavior of the system. In Figure 5 the yield stress is represented as a function of the external magnetic field. An increase of the yield stress with the external field is clearly observed. However, two different tendencies show up in the plot. For $\mathrm{H}<150-200 \mathrm{kA} / \mathrm{m}$ there is a low raise of the yield stress, whereas above this value the increase becomes sharper. 


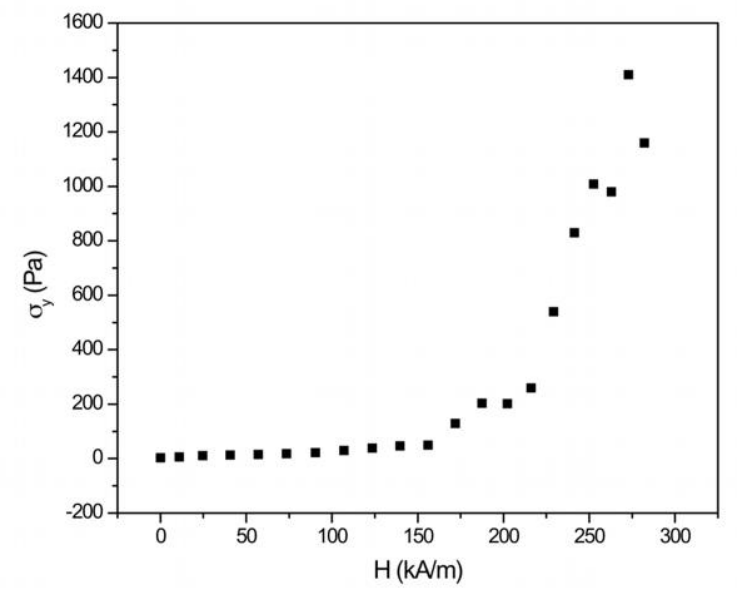

FIG 5. Static yield stress of the suspension as a function of the applied magnetic field.

However, if we observe the magnetization curve of the magnetite-covered clay particles with $\phi_{M} / \phi_{C}=3$ (Figure 1) we see that the magnetization is close to the saturation one when $H \approx 300$ $\mathrm{kA} / \mathrm{m}$. Taking into account that the curves for the highest fields have been obtained for field strengths between 252 and $282 \mathrm{kA} / \mathrm{m}$, we can suppose that for these field strengths the particles have raised a magnetization very close to the one corresponding to saturation.

The general trend of the yield stress and the plastic behavior of the suspension to increase with the external magnetic field can be semi-quantitatively explained considering the interaction energy between the particles and the formation of structures of aggregates in the suspension. The total interaction energy between the magnetite-clay composite particles is attractive (Figure 3). Then the particles form aggregates that, in the case of concentrated suspensions, span over the volume of the suspension. When the magnetic field is not applied, a small stress is enough to break the aggregates and the suspension flows just with a tiny stress value. On the other hand, when an external magnetic field is applied, the interaction energy between the particles, that is attractive, increases and the attraction between them becomes stronger. The higher the external magnetic 
field is, the more attractive the interaction energy between the particles is, and stronger the structure and higher the yield stress become. Finally, when the saturation of the material is reached, a further increase in the magnetic field does not cause a raise in the magnetic interaction energy and the yield stress does not change significantly.

The magnetic interaction energy between magnetite-covered clay particles, $V^{M A G}$, as a function of the magnetic field, calculated as explained previously is represented in Figure 6 for a surfaceto-surface distance $h=10 \mathrm{~nm}$ and for the three possible orientations between particles. It is apparent that the magnetic interaction is strongly anisotropic, the face-to-face orientation being the most favored. The magnetic interaction in the face-to-face orientation becomes much stronger than that corresponding to the other orientations as the external magnetic field is increased.

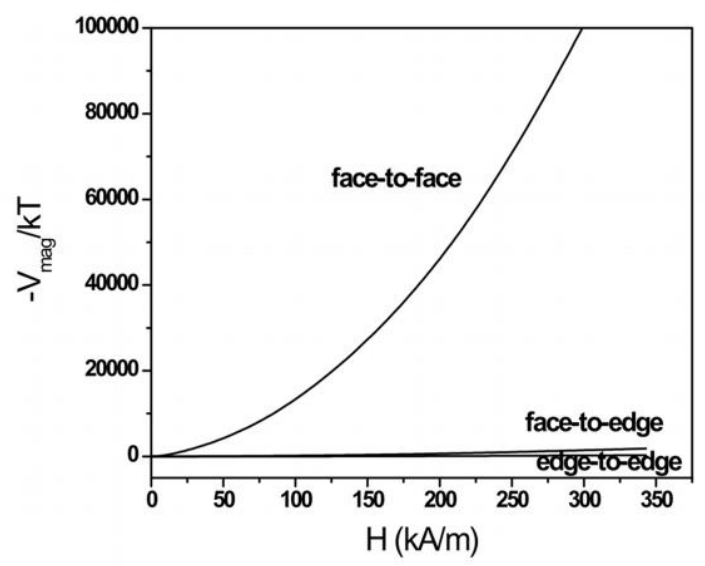

FIG 6. Magnetic interaction energy between the magnetite-clay composed particles $\left(\phi_{M} / \phi_{C}=3\right)$ as a function of the external magnetic field for the three possible orientations between particles

The increase in the magnetic interaction provokes that the structure associated to the particle aggregates becomes stiffer, and for this reason the yield stress of the suspension is higher when the external field is increased: the larger the external magnetic field, the higher the shear stress needed to break the structures and thus, to start the suspension flow. 
Nevertheless, in order to explain the change in the trend of the yield stress observed at approximately $H=150-200 \mathrm{kA} / \mathrm{m}$, the analysis of the total interaction energy between particles is required. In Figure 7 the ratio between the sum of the total interaction energy (the joint contributions of LW, EL, AB, and magnetic forces) for face-to-edge and edge-to-edge orientations relative to the total interaction energy for face-to-face orientation is represented as a function of the external magnetic field.

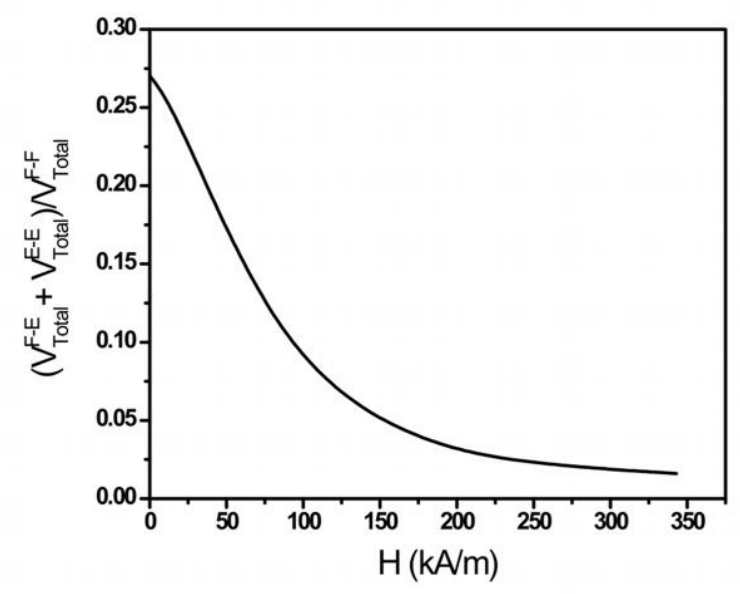

FIG 7. Ratio between the sum of the face-to-edge and edge-to-edge interaction energies and the face-to-face interaction energy as a function of the external magnetic field.

In the absence of the magnetic field the interaction energy of the face-to-edge and edge-to-edge orientations represents almost $25 \%$ of the interaction energy of the face-to-face orientation. As the magnetic field is increased this percentage becomes lower and around $150-200 \mathrm{kA} / \mathrm{m}$ it is below $5 \%$ and tends asymptotically to zero. So, when the magnetic field is not applied the contribution of face-to-face and face-to-edge interactions is lower than the face-to-face one but it cannot be neglected. The aggregates between particles in face-to-face orientation should represent the major fraction of aggregates, but some face-to-edge and edge-to-edge complexes must remain in the suspension. Hence, the structure of the particles between the plates of the rheometer can be 
considered as an ill-defined network that is stiffer the larger the magnetic field. However, when the latter reaches a value of $150-200 \mathrm{kA} / \mathrm{m}$, the face-to-face interaction becomes dominated by large, and the face-to-edge and edge-to-edge interactions become negligible. In such conditions, the structure can be imagined as a set of stacked planes (or oblate spheroids) attached to the plates of the rheometer. This structure is stronger than the initial arrangement and for this reason the increase in the yield stress with the magnetic field is more pronounced above $H \approx 150-200 \mathrm{kA} / \mathrm{m}$.

The dependence between the yield stress and the magnetic field strength can be determined considering the standard chain model proposed by Bossis et al. ${ }^{42}$ This model regards the magnetic particles as spherical and assumes that the dominant forces under shear are the hydrodynamic and magnetic ones. The permeability of the particles is the key parameter of the model, and depending on its value, one can distinguish two cases. The first one corresponds to particles of high permeability: $\alpha=\mu_{p} / \mu_{f} \gg>1, \mu_{p}$ and $\mu_{f}$ being the magnetic permeabilities of the particles and the fluid carrier, respectively. In this case, the model for the structure is based on a cubic network of infinite chains of particles aligned in the field direction. When the material is strained, the chains are supposed to deform affinely with the strain; in other words the motion of the particles takes place only along the velocity lines. The following relationship between the yield stress and the magnetic field strength is derived: ${ }^{42}$

$$
\sigma_{y}=2.31 \phi \mu_{0} M_{s}^{1 / 2} H^{3 / 2}
$$

Hence, the model predicts a linear dependence with the particle volume fraction, $\phi$, and a field dependence given by a power law $\sigma_{\mathrm{y}} \sim H^{3 / 2}$. 
The second case corresponds to particles with low permeability: $\alpha=\mu_{p} / \mu_{f}<5$. The aggregates formed in the presence of the field are either cylinders or stripes of internal volume fraction $\phi_{a}$. The yield stress is given by:

$\frac{\sigma_{y}}{\mu_{0} \mu_{f} H^{2}}=\frac{3 \sqrt{3}}{16}\left(\mu_{S}^{*}\right)^{2} \frac{\varphi(1-\varphi)}{C_{S}+\mu_{S}^{*}(1-\varphi)}$

where $\mu_{S}{ }^{*}=\left(\mu_{s} / \mu_{f}\right)-1$ is the permeability inside the aggregates; the volume fraction $\varphi=\phi / \phi_{a}$ represents the fraction of space occupied by the aggregates; $C_{S}=1$ if the particles are gathered into stripes and $C_{S}=2$ if they form cylinders. Therefore, for a given suspension, the yield stress scales with $H^{2}$.

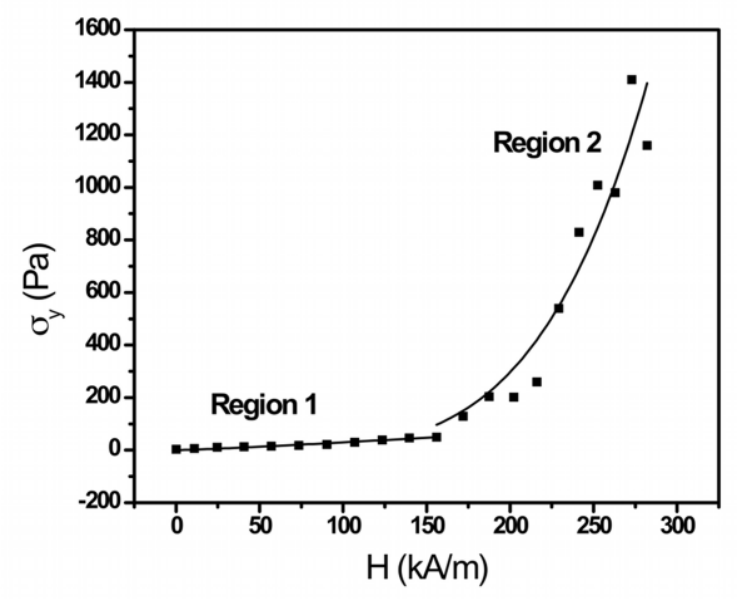

FIG 8. Fitting curves of the two regions of the yield stress as a function of the external magnetic field to the potential law $\sigma_{y}=a H^{n}$.

In our suspension of magnetite-covered clay particles, $\alpha \leq 5$ and, in principle, a scaling $\sigma_{\mathrm{y}} \sim H^{n}$ with $n \approx 2$ could be expected if the main assumptions of the model are accomplished. In order to check this hypothesis, the two separate regions in Figure 5 can be fitted to power laws of the type: $\sigma_{y}=a H^{n}$ 
The fitting curves are shown in Figure 8 and the values of the fitting parameters $(a, n)$ are displayed in Table III. The values of $n$ do not agree in neither of the cases considered with those predicted by the model.

$\underline{\text { TABLE III. Fitting parameters obtained by fitting the two regions of Figure } 8 \text { to the potential law }} \sigma_{y}=a H^{n}$

\begin{tabular}{cccc}
\hline \hline & $a$ & $n$ & $r^{2}$ \\
\hline Region 1 & $0.14 \pm 0.08$ & $1.16 \pm 0.12$ & 0.957 \\
Region 2 & $(1 \pm 5) \times 10^{-8}$ & $4.5 \pm 0.6$ & 0.918 \\
\hline \hline
\end{tabular}

This discrepancy can be explained if one takes into account that magnetite-covered units are far from spherical in shape. In spite of that, the results clearly show the remarkable difference between the values of $n$ corresponding to both regions. Taking into account that the value of the exponent in the power law is related to the particle structures formed in the suspension confined by the plates of the rheometer, this difference supports the previous hypothesis of the existence of two completely different structures in the suspension of magnetite-covered clay particles when the magnetic field is below or above $150-200 \mathrm{kA} / \mathrm{m}$.

\section{Steady state measurements. Flow regime.}

Figure 9 shows the viscosity as a function of the shear rate for the different magnetic field strengths applied. A shear thinning behavior (the viscosity decreases as the shear rate increases) is observed within the shear rate ramp applied. The magnetorheological response of the suspension can be observed as well in the figure: the viscosity for a given shear rate increases with the magnetic field strength. This increase is more appreciable when the magnetic field is above 150 $\mathrm{kA} / \mathrm{m}$ (notice the logarithm scale). This fact also supports the previous hypothesis about the change 
in the structure formed by the magnetite-clay composite particles when the magnetic field goes above $H=150 \mathrm{kA} / \mathrm{m}$.

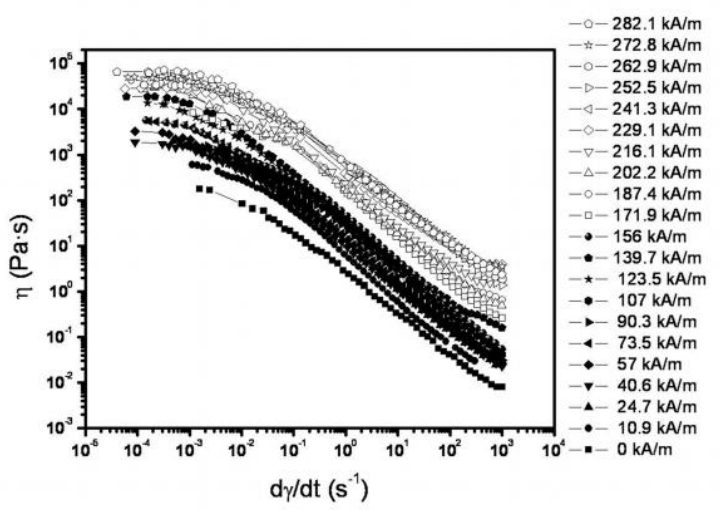

FIG 9. Viscosity of the suspension as a function of the shear rate for the different external magnetic fields applied.

In order to analyze the viscosity results, the standard chain model is applied to the experimental results. This models predicts the following relationship between the viscosity and the Mason number: ${ }^{42}$

$\eta_{F}=C M n^{-1}$

where $C=8.82$ is a constant and $\eta_{F}$ is the specific viscosity, defined as:

$\eta_{F}=\frac{\eta-\eta_{\infty}}{\phi \eta_{0}}$

being $\eta_{0}$ the viscosity of the carrier liquid, $\eta_{\infty}$ the viscosity of the suspension at very high shear rates and $\phi$ the total volume fraction of solids in the suspension.

The Mason number is the ratio between the hydrodynamic and magnetic forces: ${ }^{42}$

$M n=\frac{8 \eta_{0} \dot{\gamma}}{\mu_{0} \mu_{f} \beta^{2} H^{2}}$

where $\mu_{f}$ is the relative magnetic permeability of the carrier liquid. 
If the experimental data verify Eq. 15, it means that the magnetic forces that produce the magnetorheological effect are dipolar, and that the magnetic and hydrodynamic forces are the dominant ones. In this case, the curves of $\eta_{F}$ as a function of the Mason number should overlap in a master curve given by Eq. 15 .

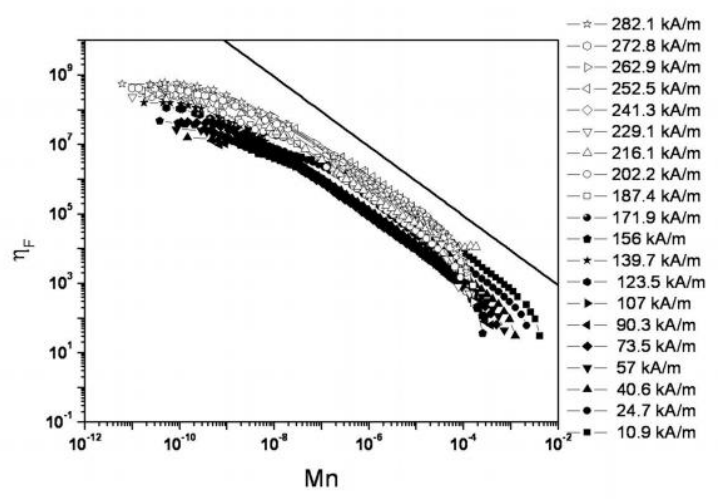

FIG 10. Specific viscosity, $\eta_{F}$ (from Eq. 16) as a function of Mason number (from Eq. 17) for different magnetic field strengths. The straight line corresponds to Eq. 15.

Figure 10 shows the specific viscosity as a function of the Mason number for the different applied magnetic field strengths. The values of $\eta_{\infty}$ have been approximated by the final data in the curves of Figure $9 ; \eta_{0} \mathrm{y} \mu_{f}$ are the viscosity $\left(10^{-3} \mathrm{~Pa} \cdot \mathrm{s}\right)$ and the relative permeability (1) of water and $\beta$ has been obtained by means of the procedure explained above (see Eq. 6 and related text). The straight line in Figure 10 corresponds to Eq. 15. We see that the curves do not overlap, although a tendency to overlap is observed at high field strength.

The absence of overlap can be explained taking into account that, at low magnetic field strengths, the hydrodynamic and magnetic forces are not the dominant ones, since the van der Waals interaction has also to be considered. As the magnetic field is increased, the magnetic interaction becomes higher as compared with the van der Waals interaction, and for this reason the curves corresponding to the highest magnetic field strengths overlap and are closer to the line 
predicted by Eq. 15. Another reason for explaining the divergence between the experimental data and the theory can be derived taking into account that the magnetite-clay composite particles are not spherical, which is another of the hypotheses of the model.

\section{E. Dynamic measurements. Oscillometry.}

With respect to the dynamic measurements, Figure 11 shows (a) the storage modulus, $G^{\prime}$, and (b) the loss modulus, $G$ ', as a function of the amplitude of the sinusoidal stress, for the different values of the applied magnetic field.

The trend of the curves is the same for the different fields: an initial plateau followed by a decreasing slope. Some of them show the beginning of a final plateau as well. The initial plateau determines the lineal viscoelastic region (LVR) of the material and provides information about the elasticity of the suspension (the higher $G$ ' versus $G$ ' 'in this region, the more elastic the suspension is, whereas a lower value of the storage modulus as compared with the loss modulus indicates a more viscous and less rigid system). The elasticity is in turn related to the particle structures in the suspension. Figure 11 shows that the amplitude of the LVR increases as the external magnetic field is increased. Hence, the suspension becomes more elastic when the magnetic field strength is increased because of the increasingly stiffer structures formed by the particles.

As mentioned above, the plateau of each curve is followed by a sharp drop. The value of the amplitude of the stress from which $G$ ' starts to fall is called critical stress, $\sigma_{c}$. The critical stress is related to the breaking of the three-dimensional field-induced structure and in the case of the curves shown in Figure 11 increases as the external magnetic field is raised. This is another confirmation of the validity of the assumption that the magnetic field strength provokes an increase in the mechanical strength of the structure. 

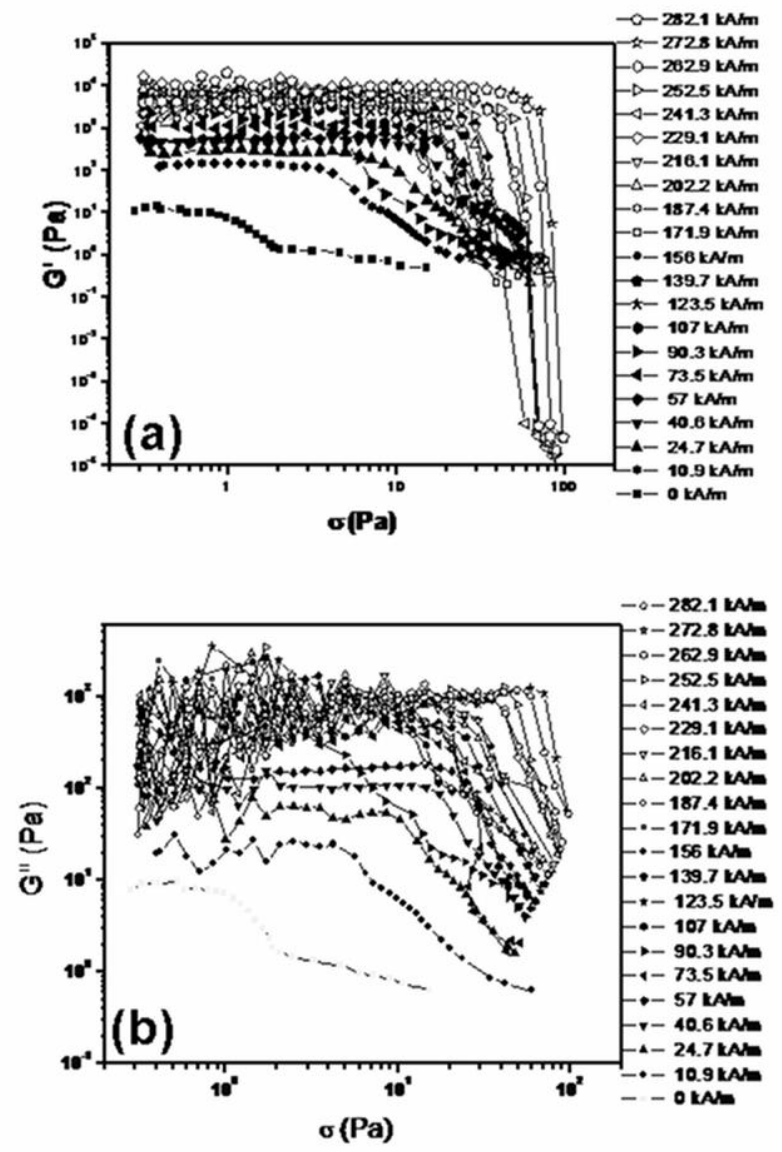

FIG 11. (a) Storage modulus, $G^{\prime}$, and (b) loss modulus, $G$ ', as a function of the amplitude of the sinusoidal stress for the suspension with $\phi_{M} / \phi_{C}=3$. The different curves correspond to the different values of the magnetic field strength shown in the legend.

In order to study these results in depth, the value of the storage modulus in the LVR (Figure 12.a) and the critical stress (Figure 12.b) are represented in Figure 12 as a function of the strength of the magnetic field. Note how both $G^{\prime}$ and $\sigma_{c}$ increase with the magnetic field strength, although their trends of variation are not monotonous within the whole magnetic field strength range. The increase in both quantities is weaker when the magnetic field is lower than $150-200 \mathrm{kA} / \mathrm{m}$ than above this value. 
As a consequence, from the oscillatory measurements it can be concluded that a magnetic field strength in the range $150-200 \mathrm{kA} / \mathrm{m}$ separates two regions with clearly differentiated mechanical properties: once this sort of critical field is reached, the field-induced structures become increasingly stronger, reaching a comparatively high mechanical strength. This agrees with our conclusions based on the steady state rheograms.
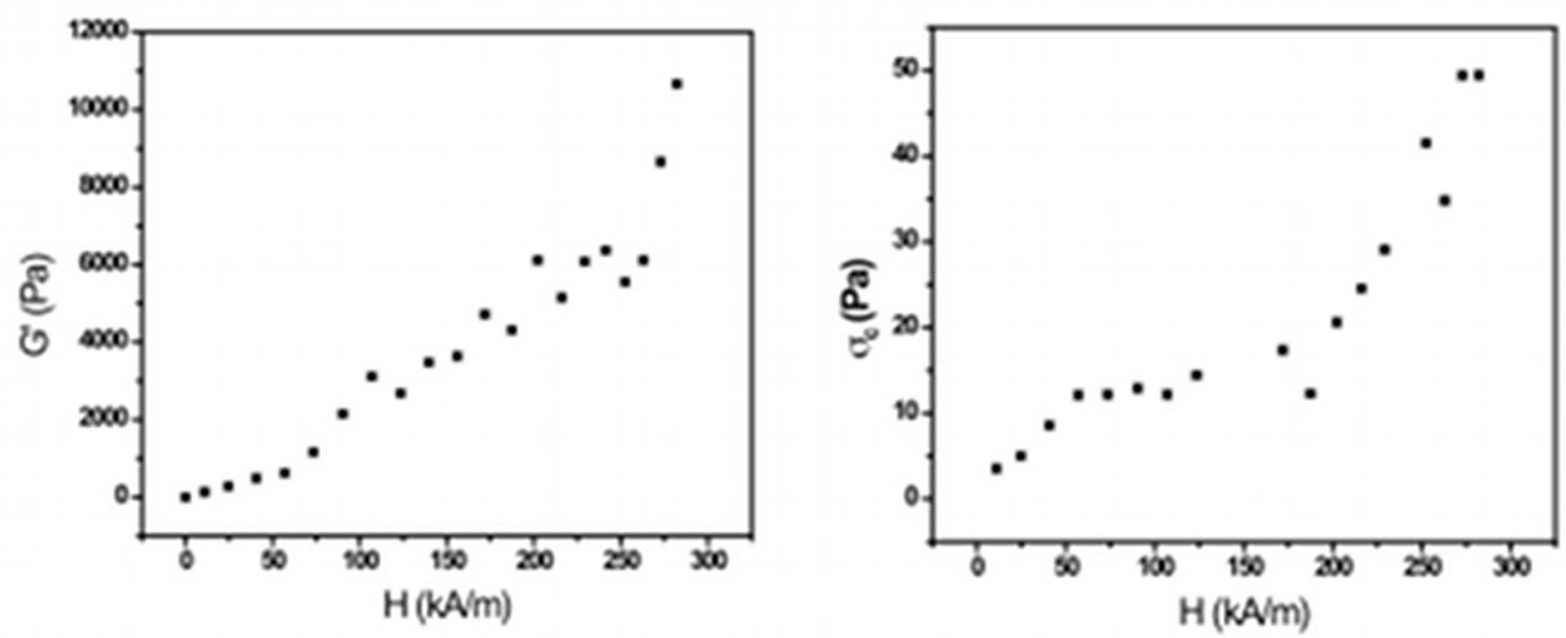

FIG 12 (a) Storage modulus $G^{\prime}$ in the LVR (plateau of the curves in Figure 11.a), and (b) critical stress $\sigma_{c}$ (value of the amplitude of the stress from which $G^{\prime}$ 'starts to fall in Figure 11.a) as a function of the external magnetic field.

\section{CONCLUSIONS}

The magnetorheological behavior of the suspensions was analyzed both at steady state regime (shear stress vs. shear rate) and in oscillatory mode (elastic and loss moduli as a function of the amplitude of the applied shear stress).

It was found that the suspensions shift from newtonian to plastic behavior when the magnetic field is applied. Interestingly, the field strength dependence of the yield stress $\left(\sigma_{y}\right)$ is not monotonous: $\sigma_{y}$ increases with $H$ much faster if the latter is above a critical value of 
approximately $150-200 \mathrm{kA} / \mathrm{m}$. The analysis of the particle-particle interactions and the geometry of the aggregates can help in elucidating the reasons for this behavior.

We propose that above that critical field, the face-to-face orientation is by large the dominant one. Consequently, the resulting stacked planes of magnetite-covered montmorillonite platelets form a stiffer structure as compared to the edge-to-edge or edge-to-face ones.

Dynamic measurements seem to confirm this hypothesis too: both the elastic modulus and the critical stress (onset of non-linear viscoelastic behavior) show a sharper increase with the field strength above the same critical magnetic field.

\section{ACKNOWLEDGMENTS}

Financial support by Ministerio de Ciencia e Innovación (Spain) under project No FIS200907321, and Junta de Andalucía (Spain) under projects No P08-FQM-3993 and No P09-FQM-4787 is gratefully acknowledged.

\section{REFERENCES}

${ }^{1}$ J. M. Ginder, Encyclopedia of Applied Physics 16, 487 (1996).

${ }^{2}$ P. P. Phulé, J. M. Ginder, MRS Bulletin 23, 19 (1998).

${ }^{3}$ J. D. Carlson and M. R. Jolly, Mechatronics, 10 (4-5), 555 (2000).

${ }^{4}$ H. See, C. Joung, C. Ekwebelam, Int J Mod Phys B, 21, 4945 (2007).

${ }^{5}$ S. Genç and P. P. Phulé, Smart Mater. Struct. 11, 140, (2002).

${ }^{6}$ A. Chaudhuri, N. M. Wereley, S. Kotha, R. Radhakrishnan and T. S. Sudarshan, J. Magn. Magn. Mater. 293, 206 (2005).

${ }^{7}$ B. D. Chin, J. H. Park, M. H. Kwon and O. O. Park, Rheol. Acta 40, 211 (2001). 
${ }^{8}$ M. T. López-López, J. de Vicente, F. González-Caballero and J.D.G. Durán, J. Colloids Surf. A, 264, 75 (2005).

${ }^{9}$ M. T. López-López, J. de Vicente, G. Bossis, F. González-Caballero and J.D.G. Durán, J. Mater. Res., 20, 874 (2005).

${ }^{10}$ P. Poddar, J. L. Wilson, H. Srikanth, J. H. Yoo, N. M. Wereley, S Kotha, L. Barghouty and R. Radhakrishnan, J. Nanosci. Nanotechnol. 4, 192, (2004).

${ }^{11}$ K. D. Weiss, D. A. Nixon, J. D. Carlson and A. J. Margida, US Patent Specification 5645752 (1997).

${ }^{12}$ N. M. Wereley, A. Chaudhuri, J. H. Yoo, S. John, S. Kotha, A. Suggs, R. Radhakrishnan, B. J. Love and T. S. Sudarshan, J. Intell. Mater. Syst. Struct. 17, 393 (2006).

${ }^{13}$ R. C. Bell, E. D. Miller, J. O. Karli, A. N. Vavreck and D. T. Zimmerman, Int. J. Mod. Phys. B 21, 5018 (2007).

${ }^{14}$ J. de Vicente, F. Vereda and J. P. Segovia-Gutiérrez, J. Rheol. 54, 1337 (2010).

${ }^{15}$ A. Gómez-Ramírez, M. T. López-López, J. D. G. Durán and F. González-Caballero, Soft Matter, 5, 3888 (2009).

${ }^{16}$ R. C. Kanu and M. T. Shaw, J. Rheol. 42, 657 (1998).

${ }^{17}$ A. Lengálová, V. Pavlínek, P. Sáha, O. Quadrat and J. Stejskal, Colloids Surf. A, 227, 1 (2003).

${ }^{18}$ M. T. López-López, G. Vertelov, P. Kuzhir, G. Bossis and J. D. G. Durán, J. Mater. Chem. 17, 3839 (2007).

${ }^{19}$ H. Nishiyama, K. Katagiri, K. Hamada, K. Kikuchi, K. Hata, P. Sang-Kyu and M. Nakano, Int. J. Mod. Phys. B, 19, 1437 (2005).

${ }^{20}$ A. Sanchis, M. Sancho, G. Martínez, J. L. Sebastián and S. Muñoz, Colloids Surf. A 249, 119 (2004). 
${ }^{21}$ J. Yin and X. Zhao, Nanotechnology, 17, 192 (2006).

${ }^{22}$ G. T. Ngatu, N. M. Wereley, J. O. Karli and R. C. Bell, Smart Mater. Struct. 17, 045022, (2008)

${ }^{23}$ C. Galindo-Gonzalez, J. De Vicente, M. M. Ramos-Tejada, M. T. Lopez-Lopez, F. GonzalezCaballero, J. D. G. Duran, Langmuir 21, 4410 (2005).

${ }^{24}$ C. Galindo-Gonzalez, G. R. Iglesias, F. Gonzalez-Caballero and J. D. G. Duran, Colloids and Surfaces A: Physicochemical and engineering aspects 306, 150 (2007).

${ }^{25}$ J. D. G. Duran, M. M. Ramos-Tejada, F. J. Arroyo and F. Gonzalez-Caballero, Journal of Colloid and Interface Science, 229 (1), 107, (2000).

${ }^{26}$ M. M. Ramos-Tejada, J. de Vicente, A. Ontiveros, J. D. G. Duran, J Rheology, 45, 1159 (2001).

${ }^{27}$ R. Massart, IEEE Trans. Magnetics 17, 1247 (1981).

${ }^{28}$ H. A. Barnes, J. F. Hutton and K. Walters, An Introduction to Rheology (Elsevier, Amsterdam, 1989).

${ }^{29}$ J. L. Viota, J. de Vicente, J. D. G. Duran, A. V. Delgado, J Colloid Interface Sci. 284, 527 (2005).

${ }^{30}$ J. de Vicente, A. V. Delgado, R. C. Plaza, J. D. G. Duran and F. Gonzalez-Caballero, Langmuir 16, 7954 (2000).

${ }^{31}$ N. W. Ashcroft and N. D. Mermin, Solid State Physics (Saunders College, Philadelphia, 1976). ${ }^{32}$ C. Galindo-Gonzalez, J. M. Feinberg, T. Kasama, L. Cervera-Gontard, M. Pósfai, I. Kósa, J. D. G. Duran, J. E. Gil, R. J. Harrison, and R. E. Dunin-Borkowski, American Mineralogist 94, 1120 (2009).

${ }^{33}$ C. J. van Oss, Interfacial Forces in Aqueous Media (Dekker, New York, 1994).

${ }^{34}$ J. Israelachivili, Intermolecular and Surface Forces (Academic Press, New York, 1992). 
${ }^{35}$ R. Hogg, T. W. Healy and D. W. Fuerstenau, J Chem. Soc. Faraday Trans. 62, 1638 (1996).

${ }^{36}$ R. A. Anderson and J. E. Martin, Am. J. Phys., 70, 11, (2002).

${ }^{37}$ J. C. M. Garnett, Philos. Trans. R. Soc. London 203, 385, (1904).

${ }^{38}$ D. Jiles, Introduction to Magnetism and Magnetic Materials (Chapman \& Hill, London, 1991).

${ }^{39}$ R. M. Bothorz, Ferromagnetism (van Nostrand, Princeton, New Jersey, 1968).

${ }^{40}$ J. A. Osborn, Physical Review E. 67, 351 (1945).

${ }^{41}$ O’Handley R.O., Modern Magnetic Materials. Principles and Application, Wiley \& Sons, New York , 2000.

${ }^{42}$ G. Bossis, O. Volkova, S. Lacis and A. Meunier, Ferrofluids (S. Odenbach, Ed Springer, Berlin, 2002) p. 202. 